\title{
Diploid vs. tetraploid Centaurium erythraea Rafn: a comparative study of regenerative in vitro potential and biosynthetic capacity
}

\author{
Biljana Filipović ${ }^{1, *}$, Branislav Šiler ${ }^{1}$, Jasmina Nestorović Žıvkovićc ${ }^{1}$, TiJana Banjanac ${ }^{1}$, \\ MariJana SKorić ${ }^{1}$, Jelena Božunović ${ }^{1}$, And DaniJela MıŠıć ${ }^{1}$ \\ ${ }^{1}$ University of Belgrade, Institute for Biological Research "Siniša Stanković" - National Institute of Republic of Serbia, Department for Plant Physiology, \\ Bulevar despota Stefana 142, 11060 Belgrade, Serbia \\ "Corresponding author: biljana.nikolic@ibiss.bg.ac.rs
}

Received: November 25, 2019

Accepted: December 12, 2019

Published on-line: December 15, 2019

Published: December 25, 2019

\begin{abstract}
The impact of ploidy level on both the regenerative potential under in vitro conditions and the production of major bioactive specialized metabolites, such as iridoids and xanthones, was examined in Centaurium erythraea Rafn. Shoot regeneration frequency was genotype dependent, but not affected by explant ploidy level. In most cases, the regenerated shoots of autotetraploid (4x) genotypes were more robust than diploid (2x) ones. Regeneration efficiency of root explants declined from the apical to the basal root segment. Shoot and root biomass production of two month-old plants was not significantly different between $2 x$ and $4 x$ genotypes. Both $4 x$ and $2 x$ genotypes were characterized by the predominance of secoiridoid glucoside gentiopicrin in shoots and roots, which is followed by sweroside and swertiamarin. Loganic acid, loganin and secologanin were much less abundant. Methylbellidifolin was the major xanthone in both shoots and roots. Diploid plants showed higher biosynthetic capacity for the production of secoiridoids and xanthones in both shoots and roots. Results highlight a higher potential of diploid C. erythraea genotypes for biotechnology-based sustainable production of secoiridoids in comparison to tetraploid genotypes.
\end{abstract}

Key words: Centaurium erythraea; diploid; autotetraploid; morphogenesis in vitro; secoiridoids; xanthones

http://dx.doi.org/10.5937/leksir1939052F

\section{INTRODUCTION}

Centaurium erythraea Rafn (common centaury), belonging to the family Gentianaceae, is well-known plant for its various important medicinal properties but also as a food or flavor additive. Aerial parts of common centaury are a rich source of different bioactive specialized metabolites, including terpenoids, phenolics, alkaloids and steroids (Šiler and Mišić, 2016). The most abundant bioactive specialized metabolites, secoiridoids and xanthones, exhibit antidiabetic, antioxidative, hepatoprotective, antibacterial, antifungal, hepatoprotective, gastroprotective, antiulcer, antiinflammatory, diuretic, antitumorigenic, analgesic, antipyretic, anti-inflammatory, antimutagenic, antirheumatic activities, and are thus commonly used for treating gastrointestinal disorders, to stimulate appetite, for regulation of digestion issues, against fever, anemia, anorexia, rheumatism, hypertension and many other conditions (reviewed in Šiler and Mišić (2016)). The dominant monoterpenoids of the iridoid type in C. erythraea are secoiridoid glycosides sweroside, swertiamarin and gentiopicrin.

Accumulation of pharmacologically valuable bioactive metabolites in plants vary due to a number of factors including changeable environmental parameters, growth conditions, genotype, different plant parts, plant development stages, etc. Polyploidy is recognized as important factor which can affect specialized metabolites production both in terms of diversity and quantities of phytochemicals. Duplication of the entire genomes is a widespread mechanism in plants which promotes evolutional success, species diversification, genetic variation and genomic complexity. Polyploids may arise through two generally recognized mechanisms: allopolyploidy, which results from interspecific hybridization and subsequent doubling of non-homologous genomes, and autopolyploidy, resulting from doubling of structurally similar, homologous genomes (Parisod et al., 2010). Autopolyploidy is more common in plants than traditionally assumed, but has received little attention compared with allopolyploidy (Parisod et al., 2010). Autopolyploidy can lead to changes in cytological, biochemical, physiological and developmental characteristics in many species, including conspicuous changes in morphology and metabolite production (Lavania, 2005; Sun et al., 2011). 
The duplicated gene dosage in polyploids offers the opportunity for increased metabolic activity/gene expression and enhanced concentration of high-value secondary metabolites in tissues and organs (Lavania, 2005). Therefore, manipulation with ploidy, as a biotechnological tool, could be useful in improving pharmaceutical value of medicinal plants, since synthetic autopolyploids usually exhibit increases in bioactive metabolite concentration compared to their diploid progenitors (Javadian et al., 2017; Kong et al., 2017; Lavania et al., 2012; Sadat Noori et al., 2017; Tavan et al., 2015; Xu et al., 2014). However, several studies have shown that polyploidization did not affect the metabolite production (Hull-Sanders et al., 2009; Wohlmuth et al., 2005) or even that the effect of polyploidy on the production of specialized metabolites was negative (Caruso et al., 2013; Cohen et al., 2013; Mansouri and Bagheri, 2017; Tan et al., 2017). Furthermore, in comparison with diploids, polyploids generally produce larger cells which can result in an increased size of plant structures such as leaves, flowers and fruit, increased biomass and often show greater resistance to biotic and abiotic stress (Lavania et al., 2012; Yildiz, 2013). The production of the synthetic polyploids is convenient because they can be compared to the diploid counterparts; however, tetraploid genotypes with natural origin can display a unique genome composition, since they have existed in natural environment for maybe hundreds of generations shaping their genetic background (Orzechowska et al., 2016).

C. erythraea is commonly found in tetraploid forms in nature (Banjanac et al., 2017; Śiler, 2012). Tetraploid populations are mainly distributed in central and northern Europe while diploid populations occurred in Mediterranean region (Mansion et al., 2005; van der Sluis, 1985). Šiler (2012) reported that among 41 randomly collected centaury populations from the Balkan Peninsula, only two populations were found as diploid. Natural populations of centaury are being disturbed by direct anthropogenic actions due to overharvesting for medicinal purposes. Modern biotechnological approaches such as in vitro culture can pose important tools for large scale plant propagation, accumulation of biomass and secondary metabolites as well as for conservation of endangered medicinal plants (Karuppusamy, 2009). Through in vitro culture of selected explants, continuous, reliable source of natural products could be provided (Caruso et al., 2013; Karuppusamy, 2009). Although centaury is characterized with extraordinary manageability in vitro and great regeneration ability (Filipović et al., 2015), to the best of our knowledge, the information on the effects of gene dosage on regeneration capacity is lacking. The aim of this study was to examine the impact of ploidy level on regenerative in vitro potential and biosynthetic capacity of Centaurium erythraea.

\section{MATERIALS AND METHODS}

\subsection{Plant material and culture conditions}

C. erythraea seeds were collected from two natural populations in locality Tjentište. The seeds of diploid population were sampled in 2007 and seeds of tetraploid population in 2016 and deposited at the Department of Plant Physiology, Institute for Biological Research "Siniša Stanković", Belgrade, Serbia, at $-20{ }^{\circ} \mathrm{C}$ until use. Surface-sterilized seeds were germinated on hormone-free half strength MS (1/2 MS) medium (Murashige and Skoog, 1962) solidified with $0.7 \%$ agar, supplemented with $3 \%$ sucrose and $100 \mathrm{mg} \mathrm{L}^{-1}$ myo-inositol and adjusted to $\mathrm{pH}$ 5.8. Ten genotypes of $2 \mathrm{x}$ and $4 \mathrm{x}$ centaury plants, with ploidy levels confirmed earlier by flow cytometry as described by Šiler (2012) and Banjanac et al. (2017), were randomly selected from in vitro pool of seedlings maintained on $1 / 2 \mathrm{MS}$. For the induction of in vitro morphogenesis, root explants, 1.0-1.5 $\mathrm{cm}$ in length, were excised either from basal, middle or apical root zone of 3 month-old plants and cultured on $1 / 2 \mathrm{MS}$ medium separately, under a 16/8 h light/dark photoperiod. Basal segments were sections in the root zone nearest to the plantlet base, while apical sections contained the root apex. For each genotype, five Petri dishes with 10 root explants each were used. The cultures were maintained at $25 \pm 2{ }^{\circ} \mathrm{C}$ under fluorescent lamps providing photon flux rate of $47 \mu \mathrm{mols}^{-1}$ $\mathrm{m}^{-2}$ the level of cultures. After 5 weeks of culturing regenerated shoots were transferred onto $1 / 2$ MS medium for rooting. Following parameters were recorded: the frequency of shoot regeneration (i.e. the percentage of explants forming shoots), the average number of shoots per root explant after 5 weeks of culturing, and shoot and root biomass of $2 x$ and $4 x$ regenerated centaury plants two months after transfering on $1 / 2 \mathrm{MS}$ medium for rooting.

\subsection{Preparation of plant extracts}

For phytochemical extraction, two month-old plantlets (shoots and roots) were ground into fine powder using liquid nitrogen About $100 \mathrm{mg}$ of plant material was extracted with $1 \mathrm{~mL}$ of 99.8\% methanol (AppliChem, Cheshire, USA), subsequently vigorously mixed by vortexing for $1 \mathrm{~min}$ and stored overnight at $4{ }^{\circ} \mathrm{C}$. After sonication for $20 \mathrm{~min}$ (RK100, Bandelin, Berlin, Germany) samples were centrifuged at $8000 \mathrm{~g}$ for $15 \mathrm{~min}$. Supernatants were filtered through $0.2 \mu \mathrm{m}$ cellulose filters (Agilent Technologies, Santa Clara, USA) and stored at $4{ }^{\circ} \mathrm{C}$ until use.

\subsection{UHPLC/DAD/MS ${ }^{2}$ quantitative analysis of targeted metabolites}

Quantification of iridoid glycosides (loganic acid, loganin), secoiridoid glycosides (secologanin, sweroside, swertiamarin, gentiopicrin) and xantones (decussatin, eustomin, methylbellidifolin, desmethyleustomin) in methanol extracts of $C$. erythraea was performed using UHPLC/DAD/MS ${ }^{2}$ analysis as previously described by Banjanac et al. (2017). Instrumentation consisted of Dionex Ultimate 3000 UHPLC system (Thermo Fisher Scientific, Bremen, Germany) with DAD and a triple quadrupole mass spectrometer (TSQ Quantum access max, Thermo Fisher Scientific, Basel, Switzerland). Samples were chromatographically separated on Hypersil gold C18 column $(50 \times 2.1 \mathrm{~mm})$ with $1.9 \mu \mathrm{m}$ particle size (Thermo FisherScientific, USA), thermostated at $40^{\circ} \mathrm{C}$. Mobile phase, flow rate and gradient elution were adjusted according to Banjanac et al. (2017), as well as heated electrospray ionization (HESI) source of the mass spectrometer. All solvents were of LCMS grade (Fisher Scientific UK, Leics, UK), and ultra-pure deionized water was generated using Water Purification System (New Human Power I Integrate, Human Corporation, Republic of Korea). The injection volume was set to $5 \mu \mathrm{L}$. All analyses were performed using three biological replicates. Targeted compounds were quantified using the selected reaction monitoring (SRM) mode of the instrument, and by tracking two diagnostic $\mathrm{MS}^{2}$ fragments of each compound. SRM chromatograms and $\mathrm{MS}^{2}$ spectra of standards are presented in Supplementary Figure 1. External standard method was used for the quantification of iridoids and xanthones, as described in Banjanac et al. (2017). Calculation of regression for each of the calibration curves showed excellent linearity with correlation coefficients of $r=0.999, p<0.001$. The total concentrations of the analyzed iridoids and xanthones were obtained by calculating peak areas and are expressed as $\mu \mathrm{g}$ per $100 \mathrm{mg}$ of plant dry weight ( $\left.\mu \mathrm{g} 100 \mathrm{mg}^{-1} \mathrm{DW}\right)$.

\subsection{Statistical analysis}

Statistical analysis was performed using Statgraphics Centurion XVI. The data regarding in vitro regeneration capacity of 

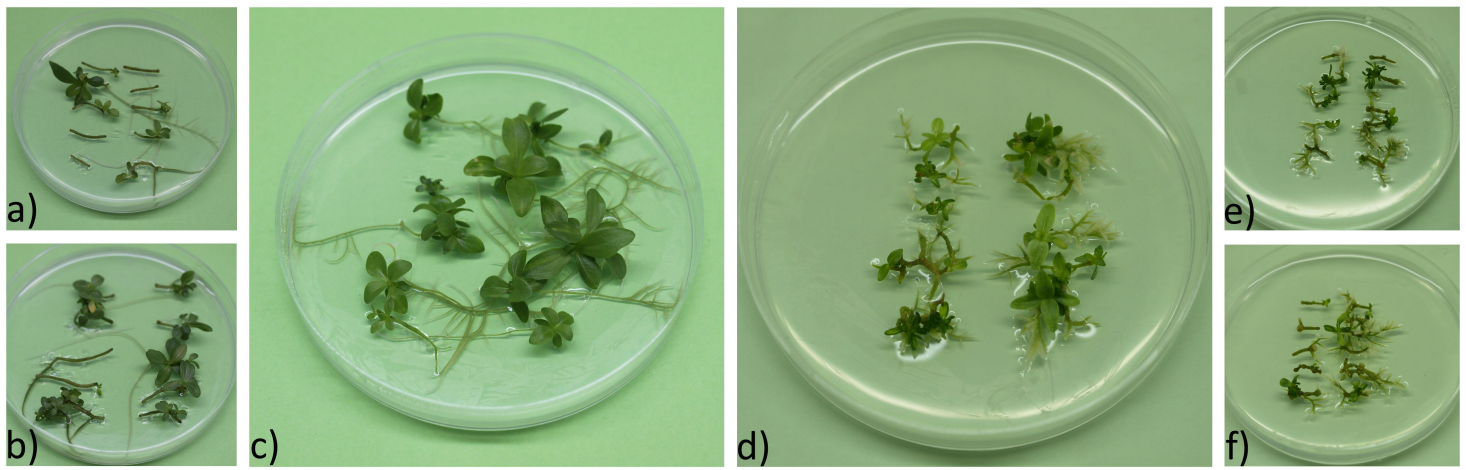

Fig. 1. Induction of morphogenesis in vitro in root culture of tetraploid and diploid plants of C. erythraea on $1 / 2 \mathrm{MS}$ medium: (a), (b), and (c) Regeneration of shoots and roots in root culture of representative tetraploid genotype 4-3; (d), (e) and (f) Regeneration of shoots and roots in root culture of diploid genotype 2-4; Regeneration was achieved using following explants: (c), (d) apical root segment; (a), (e) middle root segment; (b), (f) basal root segment

diploids and tetraploids as well as biomass of $4 x$ and $2 x$ regenerated centaury plants were analyzed by one-way ANOVA, following least significant difference test (LSD) for the confidence level of $\mathrm{P}<0.05$. Statistical analysis of the significant differences between contents of the individual compounds in diploids and tetraploids was done by the Student's $t$-test.

\section{RESULTS AND DISCUSSION}

In this study, five C. erythraea genotypes of $2 x$ and five of $4 x$ ploidy level were tested for their in vitro regeneration ability. The use of root explants for in vitro regeneration in centaury, as well as high regeneration potential of the centaury roots was described earlier (Subotić et al., 2004; 2006; Subotić and Grubišić, 2007; Trifunović-Momčilov et al., 2016). The authors showed that morphogenesis in vitro in centaury root culture is direct, without callus phase, and spontaneous, on medium without addition of any plant growth regulators (Subotić et al., 2004; Trifunović-Momčilov et al., 2016). Roots provide an excellent source of explants for mass clonal propagation because of their efficiency in the multiplication of a large number of genetically identical plants, as well as due to their easy maintenance and manipulation in vitro (Pant et al., 2010; Vinocur et al., 2000). Root cultures have also received a considerable attention due to their stable metabolite production ability (Carvalho and Curtis, 1998).

Formation of shoots on apical root explants was recorded after 5 weeks in culture (Figure 1), when $4 x$ genotypes displayed regeneration frequencies ranging between $30 \%$ and $100 \%$, while in $2 x$ genotypes this frequency was between $40 \%$ and $86 \%$ (Figure $2 \mathrm{a}$ ). The highest shoot regeneration percentage $(100 \%)$ and average shoot number per apical segment (1.7) were recorded in the tetraploid genotype $4-3$. In $2 x$ genotype $2-4$ these parameters reached $86 \%$ and 1.6. Regeneration of shoots on middle root segments varied between $14 \%$ and $72 \%$ in $4 x$ genotypes, and between $30 \%$ and $66 \%$ in $2 x$ genotypes, while basal root segments achieved regeneration frequencies between $20 \%$ and $74 \%$ in $4 x$ genotypes, and between $16 \%$ and $44 \%$ in $2 x$ genotypes. Our results indicate that regeneration frequency of centaury root explants might rather be genotype dependent (Figure 2a, 2c), but not affected by explant ploidy level (Figure 2b, 2d). This is in agreement with results of Fras and Maluszynska (2004), since calli lines, derived from the same type of explants of diploid or autotetraploid Arabidopsis thaliana plants, showed very similar regeneration ability. On the contrary, in some other species, differences in regeneration capacity under in vitro conditions between diploids and tetraploids were observed. In Dendrobium officinale regeneration ability of synthetic tetraploid cultivars was lower compared to diploid ones (Pham et al., 2019). In the last men- tioned study, the protocorm-like bodie (PLB) induction rates from the stem node of the tetraploid cultivars were significantly higher then those of diploid, but PLB proliferation of tetraploids and the mean number of plantlets regenerated from tetraploids was lower than that of the diploid. Sun et al. (2011) suggested that polyploidization caused the decrease in or loss of in vitro organogenic potential, since regeneration frequencies of shoots from leaf explants of most of the induced neopolyploids of Pyrus communis were significantly decreased compared to the original diploid control. On the contrary, few other studies showed that regeneration ability of tetraploids was increased. For example, the highest shoot regeneration percentage and shoot number per explant were obtained from petiole explants of the tetraploid line of sugar beet compared to diploid lines (Yildiz, 2013). In comparison with diploid Columbia plants, regeneration capacity of a natural tetraploid ecotype of $A$. thaliana (Warschau) was found higher under in vitro conditions (Orzechowska et al., 2016). Within the present study, clone 2-4 among diploid genotypes and clone 4-3 among tetraploid ones stand out for their regenerative potential. However, statistically significant differences between these two genotypes were observed regarding middle and basal root segments.

Obtained results showed that regeneration potential of centaury root explants was affected by the position of the isolated segment on the root (Figures 1, 2). All three types of root sections regenerated shoots, however regeneration efficiency generally declined from the apical to the basal root segment. Some genotypes were exceptions, e.g. genotype 4-1, 4-3 and 2-2 (Figures 2a, 2c). On the contrary, in Swertia hirata apical root segments remained nonresponsive, while basal and middle segments exhibited similar shoot regeneration (Pant et al., 2010). Basal segment showed the best result for producing the highest number of buds per explant in Populus tremula (Vinocur et al., 2000), middle segment was the most efficient in oriental lily cultivar "Rosato" (Kumar et al., 2008), while basal and apical segments in Brassica campestris showed the best regeneration potential (Paul and Sikdar, 2005). It is likely that centaury root explants from actively growing meristematic apical zone are better source of competent cells for shoot regeneration then sections from more differentiated root zones. Furthermore, differences in regeneration capacity may have originated from various levels of endogenous hormones in centaury root sections.

Differences in leaf size and quality of shoots regenerated from root explants of diploid and tetraploid centaury genotypes were noted. The most often regenerated shoots of $4 x$ genotypes were more robust than $2 x$ ones which also displayed hyperhydration symptomes (Figure 1). Shoots regenerated from 

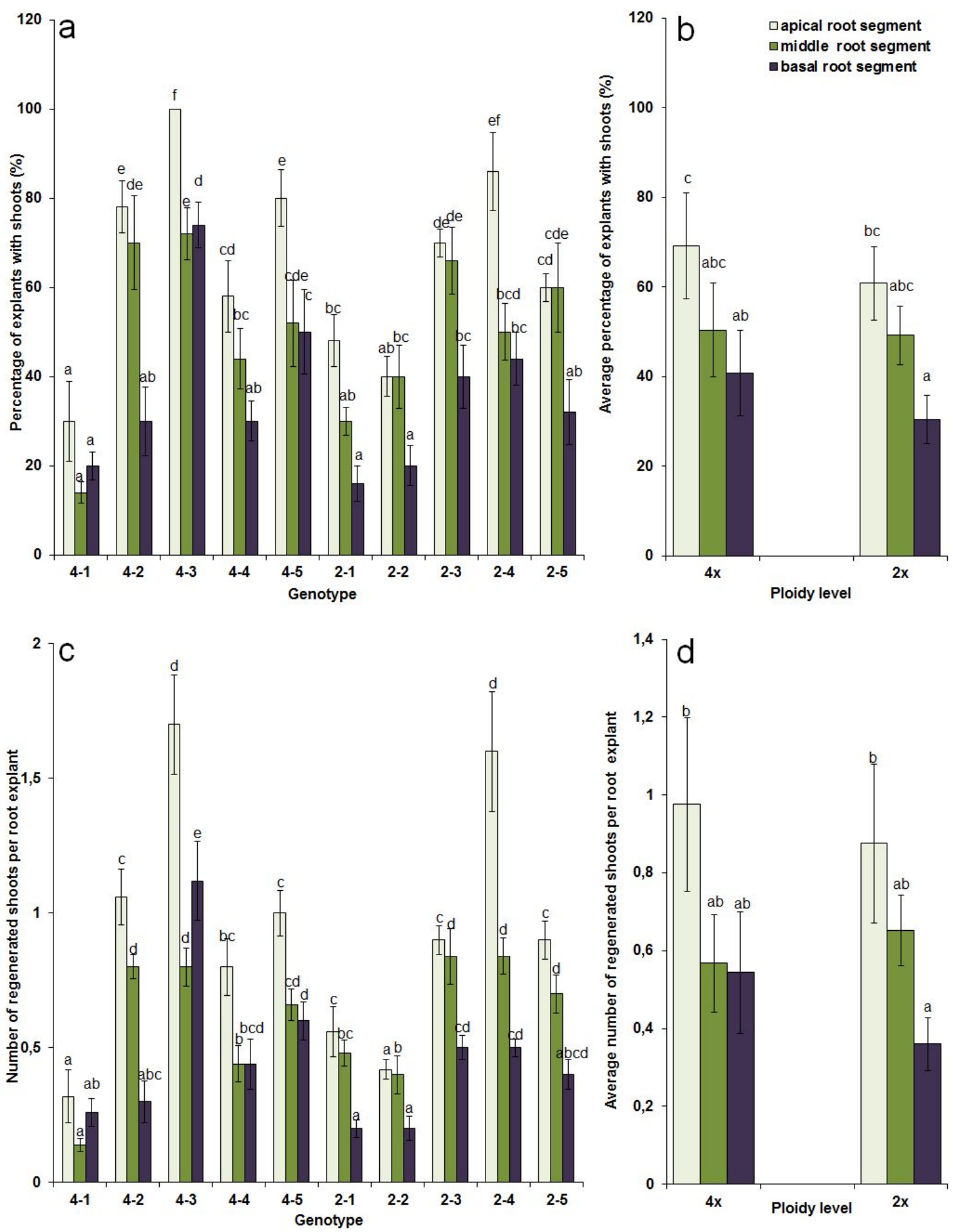

Fig. 2. Regeneration of shoots from root explants of $4 x(4-1$ to $4-5)$ and $2 x(2-1$ to $2-5)$ centaury genotypes recorded 5 weeks after culture initiation on $1 / 2$ MS hormone-free medium; (a) Percentage of explants with shoots and (c) number of regenerated shoots per explant for each genotype independently, and separately for apical (light green bars), middle (green) or basal (purple) root explants; Average values for the percentage of explants with shoots (b) and average number of regenerated shoots per root explant (d) were compared between $4 x$ and 2x genotypes. The experiment with 10 explants per Petri dish was repeated five times $(n=50)$ and results are presented as means \pm SE. Letters above the bars indicate if the values are statistically different at the $\mathrm{P}<0.05$ level according to the LSD test, independently for each type of root segments

root explants of $2 x$ and $4 x$ genotypes were successfully rooted on $1 / 2$ MS medium (Figures $3 a, 3 b, 3 c$ ). After two months in culture, $2 x$ genotypes produced higher shoot and root biomass than $4 x$ genotypes, however this difference was not statistically significant (Figure 3d). Several studies reported that tetraploid plants generally have larger organs such as leaves, flowers and fruits and higher biomass than diploids (Javadian et al., 2017; Sadat Noori et al., 2017; Xu et al., 2014). In contrast, autotetraploidy did not lead to increase in total biomasss production in maize despite its positive effect on leaf size and stem thickness (Sockness and Dudley, 1989). The overall effect of polyploidy on total biomass production is varying in different species (Abel and Becker, 2007). Biomass production of the tetraploids was even reduced in Spathiphyllum wallisii (Van Laere et al., 2011). Similarly, the effect of polyploidization on vegetative biomass production in $B$. rapa and B. oleracea was negative (Abel and Becker, 2007). Lavania et al. (2012) suggested that negative impact of polyploidy on biomass may 

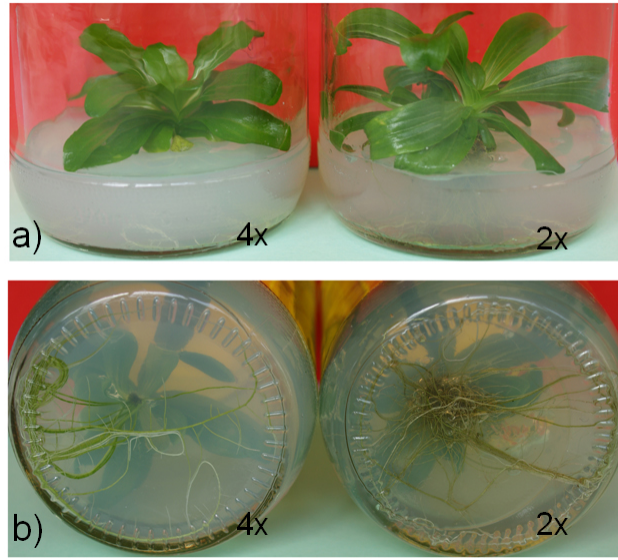
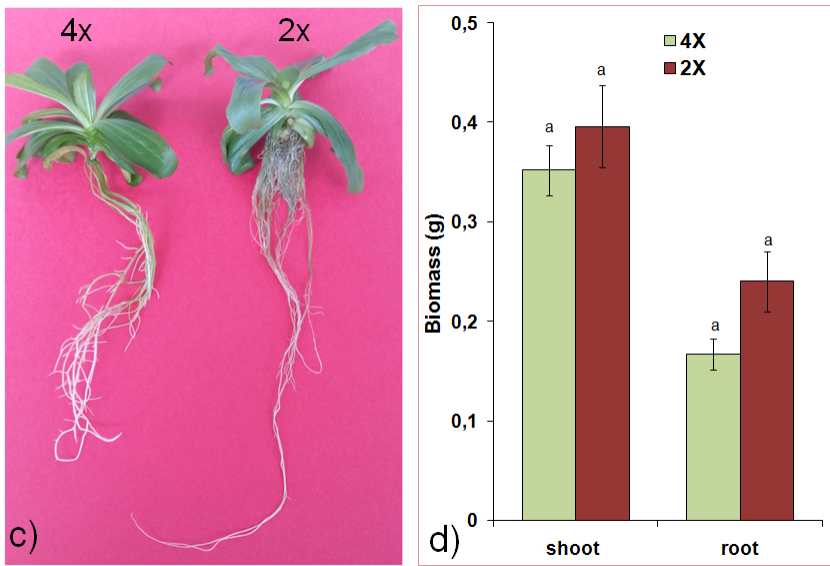

Fig. 3. Shoots (a) and roots (b) of two month-old $4 x$ and $2 x$ C. erythraea plants were removed from culture medium (c). Mean biomass (d) of shoots and roots was measured for $4 x$ and $2 x$ genotypes. Same letters above the vertical bars indicate that differences between $4 x$ and $2 x$ plants are not statistically significant at the $\mathrm{P}<0.05$ level according to the LSD test, for each plant part independently.
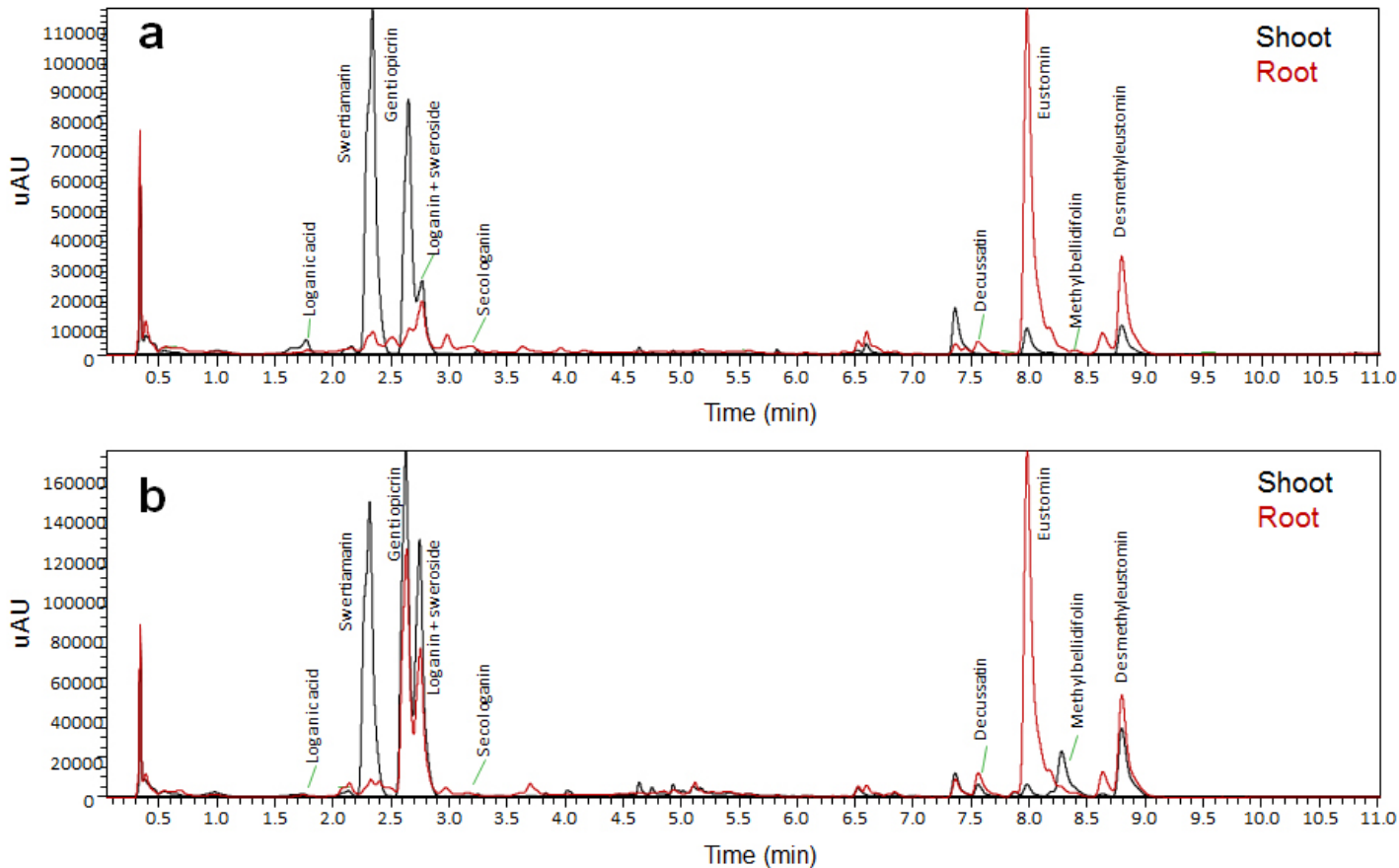

Fig. 4. UHPLC/DAD chromatograms of $4 x$ (a) and $2 x$ (b) C. erythraea genotypes, presenting major iridoids (loganic acid, loganin, secologanin, sweroside, swertiamarin and gentiopicrin) and xanthones (decussatin, eustomin, methylbellidifolin and desmethyleustomin) in shoots (black line) and roots (red line), acquired at $\lambda=260 \mathrm{~nm}$.

depend on the energy cost of produced metabolite i.e. longer the metabolic pathway larger is the negative effect of polyploidy on biomass production.

UHPLC/DAD/MS ${ }^{2}$ analysis of C. erythraea shoots and roots methanol extracts (Figure 4) was targeted towards two iridoid glycosides (loganic acid, loganin), four secoiridoid glycosides (secologanin, sweroside, swertiamarin, gentiopicrin) and four xanthones (decussatin, eustomin, methylbellidifolin, desmethyleustomin).

In both $4 x$ and $2 x$ genotypes of $C$. erythraea, the major site of targeted compounds accumulation were aerial parts (Figure 5). These results are consistent with previous findings regarding C. erythraea (Matekalo et al., 2018; Šiler et al., 2012). Both $4 \mathrm{x}$ and $2 \mathrm{x}$ genotypes of $C$. erythraea are characterized by the predominance of gentiopicrin in shoots (Figure 5a) and roots (Figure $5 b$ ). The second and third most abundant iridoids were sweroside and swertiamarin. Loganic acid, loganin and secologanin were much less abundant. Previous reports showed that the dominant secoiridoid glucoside of C. erythraea in shoots is swertiamarin (Matekalo et al., 2018; Šiler et al., 2012). Methylbellidifolin was the major xanthone in shoots and roots of both $4 x$ and $2 x$ genotypes.

In our study, diploid plants showed higher biosynthetic capacity for the production of iridoids and xanthones in both shoots and roots (Figure 5). The results indicated that the shoots of diploid plants had about 2 times higher content of gentiopicrin and sweroside than tetraploid plants (Figure 5a). Interestingly, the content of secologanin and swertiamarin were higher in shoots of tetraploid plants ( 9.48 - fold and 1.49 - fold, respectively). Similar trend was observed in roots, since the amount of gentiopicrin and sweroside were up to 1.97 times and 3.29 times higher in diploids than in tetraploids (Figure 5b). Amount of secologanin was 4.9 - fold higher in $4 x$ than in $2 x$ roots. Overall, the content of total iridoids in shoots was significantly higher in diploids, but in roots the differences were not statistically significant (Figure $5 c$ ). 

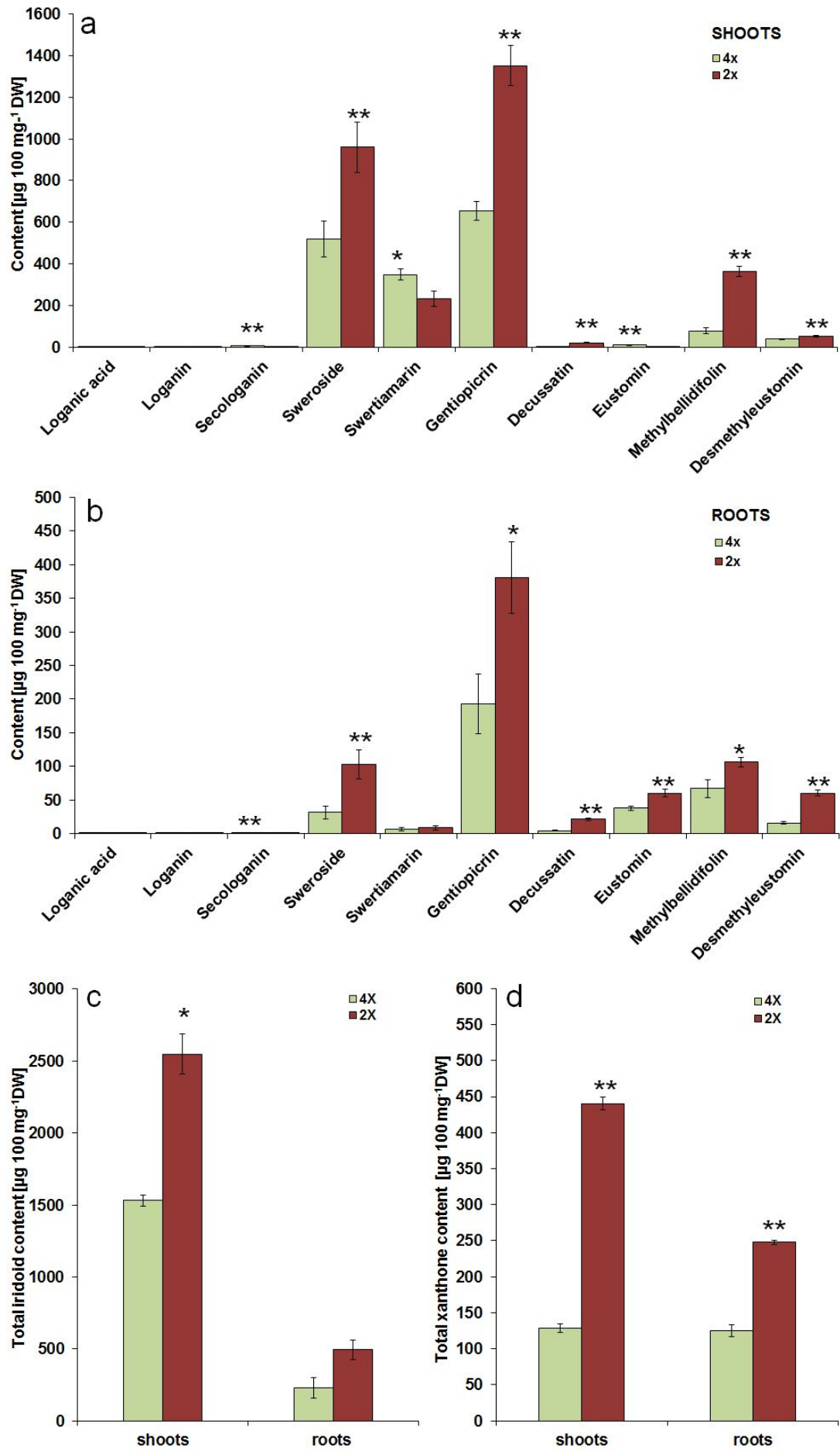

Fig. 5. UHPLC/DAD/MS ${ }^{2}$ quantification of targeted metabolites content in shoots (a) and roots $(b)$ of $4 x$ and $2 x$ regenerated centaury plants. Asterisks above the bars indicate if the values are statistically different at the $\mathrm{P}<0.05\left(^{*}\right)$ and at $\mathrm{P}<0.01\left(^{* *}\right)$ levels according to the $t$-test, for each compound independently. Total amount of monoterpenoid iridoids (c) and xanthones (d) differ significantly between $4 x$ and $2 x$ plants. Asterisks above the bars indicate if the values are statistically different at the $\mathrm{P}<0.05{ }^{*}$ ) and at $\mathrm{P}<0.01\left(^{* *}\right)$ levels according to the $t$-test, for each plant part independently. Abbreviations: DW - dry weight.

The content of total xanthones in shoots and roots of diploid plants was significantly higher than in tetraploid plants (Figure 5d). Higher content of decussatin, methylbellidifolin and desmethyleustomin were observed in shoots of diploid plants than in tetraploid plants. The exception was eustomin, with content 2.3 - fold higher in $4 x$ than in 2x plants (Figure 5a). The content of decussatin, eustomin, methylbellidifolin and desmethyleustomin in $2 x$ roots were 4.8 - fold, 1.6 - fold, 1.6 fold and 3.78 - fold higher compared to those of $4 x$ roots, respectively (Figure $5 \mathrm{~b}$ ). Literature survey pointed to the studies which explained that polyploidization in plants results either in increases or decreases in specialized metabolites production, depending on the species in question. For example, concentrations of gingerol were lower for the tetraploid clones than for the parent diploid Zingiber officinale, although the differences were not statistically significant (Wohlmuth et al., 2005). Betacyanins, the major pigments in fruit of diploid Hylocereus monacanthus and synthetic allotriploid, decreased following genome doubling (Cohen et al., 2013). Similarly, tetraploid genotypes of Solanum bulbocastanum displayed either similar or lower amounts of phenylpropanoids, tryptophan, tyrosine and $\alpha$-chaconine compared to diploids (Caruso et al., 2013) Furthermore, non-targeted and targeted metabolic profiling of mature leaves from three doubled diploids of citrus and their 
diploid controls showed that primary metabolism takes priority over specialized metabolism in doubled diploid plants, since the levels of primary metabolites tended to increase and the levels of secondary metabolites tended to decrease (Tan et al., 2017). As can be observed, there is a lack of linearity between the increase in genetic material (ploidy level) and the production rate of specialized metabolites in different medicinal and aromatic plants (Iannicelli et al., 2020). Changes in the metabolic profile of autopolyploids were interpreted as being the result of an alteration in the mechanism(s) regulating the biosynthesis of individual compounds (Fasano et al., 2016). Naturally occuring polyploids have been under pressures of the natural selection for a long period of time after polyploidization and therefore differences in metabolite concentration between diploid and polyploid genotypes may be due to selection. In the Balkan Peninsula tetraploid populations of C. erythraea dominate over diploid ones, which are recorded in only two locations (Šiler et al., 2012). The wide distribution of tetraploids in common centaury implies that this genome status has an adaptive significance and that it is selected positively. Secondary metabolites are often produced to protect plants against biotic and abiotic stresses and require a high investment in energy and carbon (Tan et al., 2017). Taking into account that C. erythraea plants within the present study were grown under experimental in vitro conditions where $2 x$ and $4 x$ genotypes were exposed to the same environment, the differences in specialized metabolites production may be attributed to genetic differences between the two groups of samples, and to their metabolic capacity under these specific conditions.

\section{CONCLUSION}

Our results highlighted a great potential of diploid C. erythraea genotypes for biotechnology-based sustainable production of secoiridoids. Higher production of secoiridoids and xanthones in shoots and roots of diploid centaury plants regenerated in vitro, as well as their successful regeneration from root explants, indicate a great potential of in vitro cultivation of diploid centaury plants for obtaining valuable bioactive metabolites on a large scale. Such cultures may therefore provide an alternative to naturally grown plants as a potential biomass source for the production of bioactive compounds. Further studies are conducted towards elucidation of molecular background of secoiridoid glycosides production as influenced by ploidy level.

\section{ACKNOWLEDGMENTS}

This work was financially supported by the Serbian Ministry of Education, Science and Technological Development (grant No. OI173024).

\section{REFERENCES}

Abel, S. and Becker, H. C. (2007). The effect of autopolyploidy on biomass production in homozygous lines of Brassica rapa and Brassica oleracea, Plant Breeding 126(6): 642-643.

Banjanac, T., Dragićević, M., Šiler, B., Gašić, U., Bohanec, B., Nestorović Živković, J., Trifunović, S. and Mišić, D. (2017). Chemodiversity of two closely related tetraploid Centaurium species and their hexaploid hybrid: Metabolomic search for high-resolution taxonomic classifiers, Phytochemistry 140: 2744.

Caruso, I., Dal Piaz, F., Malafronte, N., De Tommasi, N., Aversano, R., Zottele, C. W., Scarano, M.-T. and Carputo, D. (2013). Impact of ploidy change on secondary metabolites and photochemical efficiency in Solanum bulbocastanum, Natural Product Communications 8(10): 1934578X1300801.

Carvalho, E. B. and Curtis, W. R. (1998). Characterization of fluid-flow resistance in root cultures with a convective flow tubular bioreactor, Biotechnology and Bioengineering 60(3): 375-384.

Cohen, H., Fait, A. and Tel-Zur, N. (2013). Morphological, cytological and metabolic consequences of autopolyploidization in Hylocereus (Cactaceae) species, BMC Plant Biology 13(1): 173.

Fasano, C., Diretto, G., Aversano, R., D'Agostino, N., Di Matteo, A., Frusciante, L., Giuliano, G. and Carputo, D. (2016). Transcriptome and metabolome of synthetic Solanum autotetraploids reveal key genomic stress events following polyploidization, New Phytologist 210(4): 1382-1394.

Filipović, B. K., Simonović, A. D., Trifunović, M. M., Dmitrović, S. S., Savić, J. M., Jevremović, S. B. and Subotić, A. R. (2015). Plant regeneration in leaf culture of Centaurium erythraea Rafn. Part 1: The role of antioxidant enzymes, Plant Cell, Tissue and Organ Culture (PCTOC) 121(3): 703-719.

Fras, A. and Maluszynska, J. (2004). The correlation between the chromosome variation in callus and genotype of explants of Arabidopsis thaliana, Genetica 121(2): 145-154.

Hull-Sanders, H. M., Johnson, R. H., Owen, H. A. and Meyer, G. A. (2009). Effects of polyploidy on secondary chemistry, physiology, and performance of native and invasive genotypes of Solidago gigantea (Asteraceae), American Journal of Botany 96(4): 762-770.

Iannicelli, J., Guariniello, J., Tossi, V., Regalado, J., Di Ciaccio, L., van Baren, C., Pitta Álvarez, S. and Escandón, A. (2020). The "polyploid effect" in the breeding of aromatic and medicinal species, Scientia Horticulturae 260: 108854.

Javadian, N., Karimzadeh, G., Sharifi, M., Moieni, A. and Behmanesh, M. (2017). In vitro polyploidy induction: changes in morphology, podophyllotoxin biosynthesis, and expression of the related genes in Linum album (Linaceae), Planta 245(6): 1165-1178.

Karuppusamy, S. (2009). A review on trends in production of secondary metabolites from higher plants by in vitro tissue, organ and cell cultures, Journal of Medicinal Plants Research 3(13): 1222-1239.

Kong, D., Li, Y., Bai, M., Deng, Y., Liang, G. and Wu, H. (2017). A comparative study of the dynamic accumulation of polyphenol components and the changes in their antioxidant activities in diploid and tetraploid Lonicera japonica, Plant Physiology and Biochemistry 112: 87-96.

Kumar, S., Chaudhary, V. and Kanwar, J. K. (2008). Bulblet regeneration from in vitro roots of oriental lily hybrid, Journal of Fruit and Ornamental Plant Research 16: 8.

Lavania, U. (2005). Genomic and ploidy manipulation for enhanced production of phyto-pharmaceuticals, Plant Genetic Resources 3(2): 170-177.

Lavania, U. C., Srivastava, S., Lavania, S., Basu, S., Misra, N. K. and Mukai, Y. (2012). Autopolyploidy differentially influences body size in plants, but facilitates enhanced accumulation of secondary metabolites, causing increased cytosine methylation, The Plant Journal 71(4): 539-549. 
Mansion, G., Zeltner, L. and Bretagnolle, F. (2005). Phylogenetic patterns and polyploid evolution within the Mediterranean genus Centaurium (Gentianaceae - Chironieae), TAXON 54(4): 931-950.

Mansouri, H. and Bagheri, M. (2017). Induction of polyploidy and its effect on Cannabis sativa L., in S. Chandra, H. Lata and M. A. ElSohly (eds), Cannabis sativa L. - Botany and Biotechnology, Springer International Publishing, Cham, pp. 365-383.

Matekalo, D., Skorić, M., Nikolić, T., Novaković, L., Lukić, M., Božunović, J., Aničić, N., Filipović, B. and Mišić, D. (2018). Organ-specific and genotype-dependent constitutive biosynthesis of secoiridoid glucosides in Centaurium erythraea Rafn, and its elicitation with methyl jasmonate, Phytochemistry 155: 69-82.

Murashige, T. and Skoog, F. (1962). A revised medium for rapid growth and bio assays with tobacco tissue cultures, Physiologia Plantarum 15(3): 473-497.

Orzechowska, M., Gurdek, S., Siwinska, D. and PiekarskaStachowiak, A. (2016). Cytogenetic characterization of the Arabidopsis thaliana natural tetraploid ecotype Warschau stability during in vitro regeneration, Plant Cell, Tissue and Organ Culture (PCTOC) 126(3): 553-560.

Pant, M., Bisht, P. and Gusain, M. P. (2010). De novo shoot organogenesis from cultured root explants of Swertia chirata Buch.-Ham.ex Wall.: an endangered medicinal plant., Nature and Science 8(9): 244-252.

Parisod, C., Holderegger, R. and Brochmann, C. (2010). Evolutionary consequences of autopolyploidy: Research review, New Phytologist 186(1): 5-17.

Paul, S. and Sikdar, S. R. (2005). Regeneration of plants from root explant of two Indian cultivars of Brassica campestris L. through somatic embryogenesis, Current Science 89(8): 13231326.

Pham, P.-L., Li, Y.-X., Guo, H.-R., Zeng, R.-Z., Xie, L., Zhang, Z.-S., Chen, J., Su, Q.-L. and Xia, Q. (2019). Changes in Morphological Characteristics, Regeneration Ability, and Polysaccharide Content in Tetraploid Dendrobium officinale, HortScience 54(11): 1879-1886.

Sadat Noori, S. A., Norouzi, M., Karimzadeh, G., Shirkool, K. and Niazian, M. (2017). Effect of colchicine-induced polyploidy on morphological characteristics and essential oil composition of ajowan (Trachyspermum ammi L.), Plant Cell, Tissue and Organ Culture (PCTOC) 130(3): 543-551.

Šiler, B. (2012). Phytochemical and molecular characterization of centaury (Centaurium erythraea Rafn.) populations from the Balkan Peninsula, PhD thesis, University of Belgrade, Begrade, Serbia.

Šiler, B., Avramov, S., Banjanac, T., Cvetković, J., Nestorović Živković, J., Patenković, A. and Mišić, D. (2012). Secoiridoid glycosides as a marker system in chemical variability estimation and chemotype assignment of Centaurium erythraea Rafn from the Balkan Peninsula, Industrial Crops and Products 40: 336-344.

Šiler, B. and Mišić, D. (2016). Biologically active compounds from the genus Centaurium s.l. (Gentianaceae), Studies in Natural Products Chemistry, Vol. 49, Elsevier, pp. 363-397.

Sockness, B. and Dudley, J. W. (1989). Morphology and yield of isogenic diploid and tetraploid maize iinbreds and hybrids, Crop Science 29(4): 1029.
Subotić, A., Budimir, S., Grubišić, D. and Momčilović, I. (2004) Direct regeneration of shoots from hairy root cultures of Centaurium erythraea inoculated with Agrobacterium rhizogenes, Biologia Plantarum 47(4): 617-619.

Subotić, A. and Grubišić, D. (2007). Histological analysis of somatic embryogenesis and adventitious shoot formation from root explants of Centaurium erythreae Gillib, Biologia Plantarum 51(3): 514-516.

Subotić, A., Janković, T., Jevremović, S. and Grubišić, D. (2006). Plant tissue culture and secondary metabolites productions of Centaurium erythraea rafn., a medicinal plant, in T. da Silva JA (ed.), Floriculture ornamental and plant biotechnology: advances and topical issues, $1^{\text {st }}$ edn, Vol. $2^{\text {nd }}$, Global Science Books, London, pp. 564-570.

Sun, Q., Sun, H., Bell, R. L., Li, H. and Xin, L. (2011). Variation of phenotype, ploidy level, and organogenic potential of in vitro regenerated polyploids of Pyrus communis, Plant Cell, Tissue and Organ Culture (PCTOC) 107(1): 131-140.

Tan, F.-Q., Tu, H., Wang, R., Wu, X.-M., Xie, K.-D., Chen, J.J., Zhang, H.-Y., Xu, J. and Guo, W.-W. (2017). Metabolic adaptation following genome doubling in citrus doubled diploids revealed by non-targeted metabolomics, Metabolomics 13(11): 143.

Tavan, M., Mirjalili, M. H. and Karimzadeh, G. (2015). In vitro polyploidy induction: changes in morphological, anatomical and phytochemical characteristics of Thymus persicus (Lamiaceae), Plant Cell, Tissue and Organ Culture (PCTOC) 122(3): 573-583.

Trifunović-Momčilov, M., Motyka, V., Dragićević, I. ., Petrić, M., Jevremović, S., Malbeck, J., Holík, J., Dobrev, P. I. and Subotić, A. (2016). Endogenous phytohormones in spontaneously regenerated Centaurium erythraea Rafn. plants grown in vitro, Journal of Plant Growth Regulation 35(2): 543552.

van der Sluis, W. G. (1985). Chemotaxonomical investigations of the genera Blackstonia and Centaurium (Gentianaceae), Plant Systematics and Evolution 149(3): 253-286.

Van Laere, K., França, S. C., Vansteenkiste, H., Van Huylenbroeck, J., Steppe, K. and Van Labeke, M.-C. (2011). Influence of ploidy level on morphology, growth and drought susceptibility in Spathiphyllum wallisii, Acta Physiologiae Plantarum 33(4): 1149-1156.

Vinocur, B., Carmi, T., Altman, A. and Ziv, M. (2000). Enhanced bud regeneration in aspen (Populus tremula L.) roots cultured in liquid media, Plant Cell Reports 19(12): 11461154 .

Wohlmuth, H., Leach, D. N., Smith, M. K. and Myers, S. P. (2005). Gingerol content of diploid and tetraploid clones of ginger (Zingiber officinale Roscoe), Journal of Agricultural and Food Chemistry 53(14): 5772-5778.

Xu, C.-g., Tang, T.-x., Chen, R., Liang, C.-h., Liu, X.-y., Wu, C.-1., Yang, Y.-s., Yang, D.-p. and Wu, H. (2014). A comparative study of bioactive secondary metabolite production in diploid and tetraploid Echinacea purpurea (L.) Moench, Plant Cell, Tissue and Organ Culture (PCTOC) 116(3): 323-332.

Yildiz, M. (2013). Plant responses at different ploidy levels, in M. Silva-Opps (ed.), Current Progress in Biological Research, InTech. 